\title{
Chemical microscopy and nanoscopy of bio-materials and living cells
}

\author{
Erik Bründermann ${ }^{\mathrm{a}, \mathrm{i}}$, D. A. Schmidt ${ }^{\mathrm{a}}$, I. Kopf ${ }^{\mathrm{a}}$, K. Meister ${ }^{\mathrm{a}}$, M. Filimon ${ }^{\mathrm{a}}$, and M. Havenith ${ }^{\mathrm{a}}$ \\ ${ }^{a}$ Physikalische Chemie II, Ruhr-Universität Bochum, 44780 Bochum, Germany
}

\begin{abstract}
Raman microspectroscopy of human sperm cells and nearfield infrared microscopy of nanoscale biomaterials like nanografted DNA have been performed. A newly developed station for the ANKA-IR2 beamline combines several techniques to study the same sample at the same position.
\end{abstract}

\section{INTRODUCTION AND BACKGROUND}

$\mathrm{I}$ MAGING with microscale and even nanoscale resolution combined with infrared and terahertz spectroscopy can reveal processes in living cells in a label-free manner. As part of the development of a new experimental station for the ANKA-IR2 beamline in Karlsruhe, we have combined scattering scanning near-field infrared microscopy (s-SNIM) $)^{1,2}$ with other techniques like confocal Raman microspectroscopy $(\mathrm{CRM})^{3}$, atomic force microscopy (AFM) and scanning optical near-field microscopy (SNOM) in a single instrument. This combination holds the potential to investigate the same sample at the same position with different, yet complementary techniques which are sensitive to the chemistry (CRM, sSNIM), mechanical properties (AFM), crystallinity (CRM) and to the influence of fluorophors (SNOM) at a variety of length scales from micrometers to nanometers.

\section{RAMAN MICRO-SPECTROSCOPY}

Figure 1 shows the instrument for the experimental station at the ANKA-IR2 beamline.

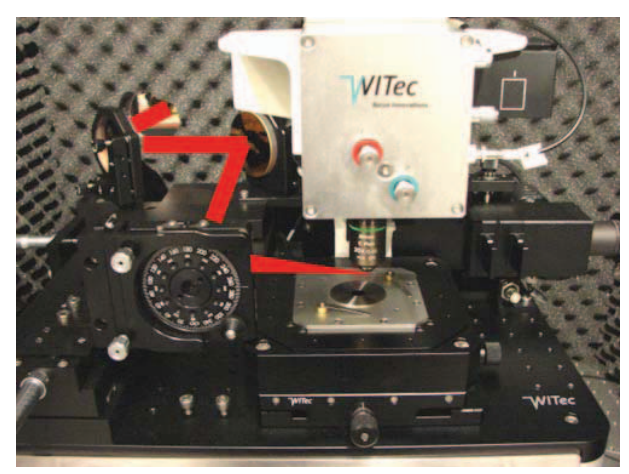

Fig. 1: Photo of microscope (WITec GmbH, Germany, combined model alpha300A, 300S, and 300R) augmented with s-SNOM for using external light sources such as lasers or synchrotron radiation. An off-axis parabolic mirror focuses the light onto a metalized AFM nano-tip. The scattered light is detected in the far-field but contains near-field information of the sample with a spatial resolution determined by the curvature radius of the tip, typically 30 to $40 \mathrm{~nm}$.

It combines an AFM, a CRM, aperture-based SNOM, and,

${ }^{i}$ Honorable Guest Professor, Dept. of Nanovision Technology, Shizuoka University, Hamamatsu, Japan in addition, s-SNOM for infrared and $\mathrm{THz}$ radiation. Measurements of the "same" sample at the "same" position with different microscopy modes can give new insights, especially, in heterogeneous biomaterials.

Recently, we used CRM to investigate human sperm cells $\mathrm{s}^{3}$ and to reconstruct chemical maps from the composition of the sub-cellular compartments (see Fig. 2). Raman mapping allowed to unambiguously characterize the nucleus, the neck, and, in particular, the mitochondria-rich middle piece of a human sperm cell. The effect of UV radiation on different organelles of the sperm was quantified by localized spectral Raman signatures obtained within milliseconds. Chemical changes within the sub-cellular structure of the sperm cells were recorded as a function of UV light exposure time. The study showed that Raman microspectroscopy can be a fast diagnostic method for detecting the mitochondrial and motility status of human spermatozoa ${ }^{3}$ as recognized in "Highlights in Chemical Biology" of the Royal Society of Chemistry.

The sub-micron resolution of confocal Raman microspectroscopy was a prerequisite to localize fast chemical changes in the middle piece.

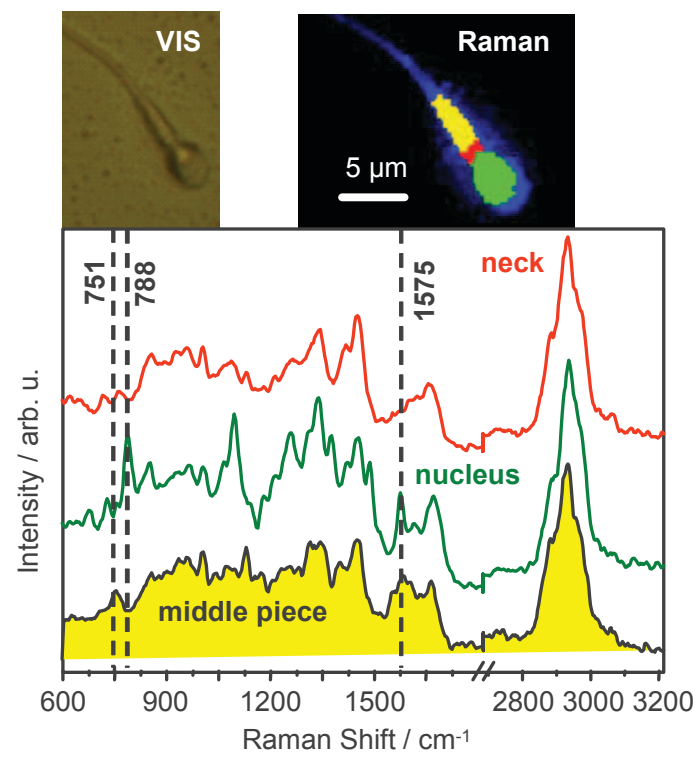

Fig. 2: The upper part shows an optical image (VIS) of a human sperm cell and the chemical map constructed from Raman measurements taken on the single cell. A scale bar of $5 \mu \mathrm{m}$ is indicated. The chemical image is based upon a k-means cluster analysis from $400-3400 \mathrm{~cm}^{-1}$. The nucleus (green), neck (red) and the middle piece (yellow) are identified ${ }^{3}$.

\section{NEAR-FIELD MICROSCOPY OF BIOMATERIALS}

SNIM, scattering SNOM at infrared frequencies, can further 
increase the spatial resolution by a factor of up to 100 . We developed for the ANKA-IR2 beamline a new experimental station which combines CRM and SNIM in a single instrument with the possibility to investigate the same sample at the same position with complementary techniques. By laserbased SNIM, we have investigated biomaterials like nanografted DNA (Fig. 3) allowing nano-spectroscopy of only a few hundred DNA molecules ${ }^{4}$. A conservative estimate considers a detection area defined by $2 R$, with $R$ the nano-tip radius of curvature. The number of double-stranded (ds)-DNA molecules in an area of $(125 \mathrm{~nm})^{2}$ is approximately 120 , which is 1.5 attogram. A single cell contains approximately a factor of $10^{5}$ more nucleic acid mass. This high sensitivity promises the possibility to harvest and detect DNA of a single cell without the need for polymerase chain reactions (PCR). PCR is typically used to amplify a single or few copies of DNA and to increase the detection sensitivity by using higher concentrations.

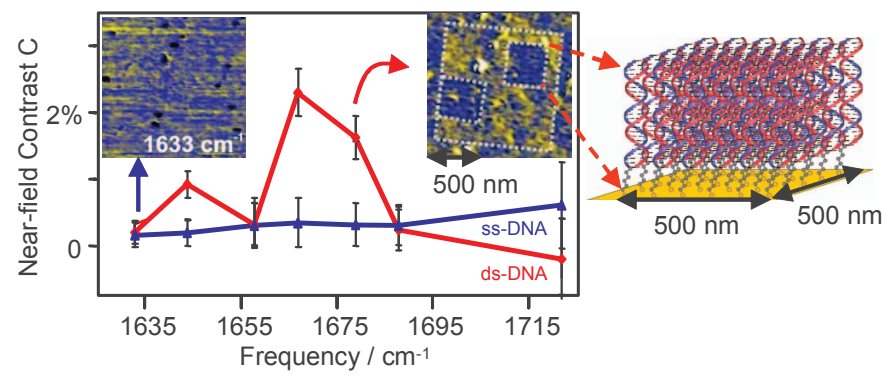

Fig. 3: Frequency dependent near-field contrast $\mathrm{C}$ of a few hundred DNA molecules for nanografted double-stranded (ds)-DNA and single-stranded (ss)-DNA ${ }^{4}$. A change in contrast can be found upon hybridization. Near-field images for 1679 and $1633 \mathrm{~cm}^{-1}$ are depicted. The small blue nanografted squares have a size of approximately $500 \mathrm{~nm}$ (on the right, see illustration of ds-DNA linked to the gold surface). Contrast of the small squares is visible if the laser coincides with absorption of DNA upon hybridization.

\section{NEAR-FIELD MICROSCOPY OF NANO-MATERIALS}

Materials like "single" nanoparticles formed in dusty plasmas $^{5}$ show evidence for different growth mechanisms as a function of time. SNIM, infrared nano-spectroscopy, of single nanoparticles are not prone to low signal-to-noise ratios because the values are not averaged over a large ensemble. The ensemble particles can vary in dimension due to different growth conditions resulting from fluctuations of system parameters such as particle density and electric field inhomogeneity, during the formation of plasma particles. These variations can wash out effects which are visible with a high signal-to-noise ratio for each individual nano-particle.

Polymers, and so-called polymer brushes, can provide new functional materials, but it is difficult to distinguish different polymer brushes with nano-scale dimensions using noninvasive techniques. SNIM is a suitable method to detect various polymer brushes on the nano-scale . $^{6}$

The instrument as well as chemical microscale and nanoscale mapping will be presented.

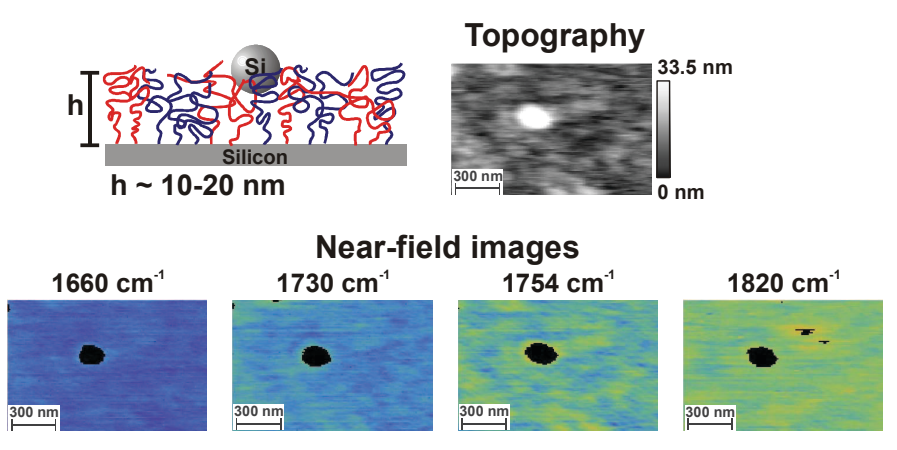

Fig. 4: Frequency dependent near-field infrared contrast $\mathrm{C}$ of a PS-PMMA mixed polymer brush ${ }^{6}$. The lateral scale bar is $300 \mathrm{~nm}$. PMMA rich domains can be distinguished due to the specific response of its carboxyl group around $1750 \mathrm{~cm}^{-1}$. Si nanoparticles were used as a reference due to their nearly frequency-independent near-field response in the investigated infrared spectral range.

\section{ACKNOWLEDGMENT}

We acknowledge support by grant BMBF 05KS7PC2 for innovative instrumentation of the synchrotron ANKA and 05K10PCA. We thank B. Gasharova, Y.-L. Mathis, A.-S. Müller, and the ANKA-team for numerous discussions. I. Kopf acknowledges financial support by SMD. Single Molecule Detection (SMD) is a project funded by the EU Commission under the 7th Framework Programme. M. Filimon acknowledges financial support by Marie Curie Early Stage Research Training, in the framework of the project INTCHEM (MEST-CT-2005-020681).

\section{REFERENCES}

[1] F. Keilmann, R. Hillenbrand, Near-field nanoscopy by elastic light scattering from a tip, in Nano-Optics and Near-Field Optical Microscopy, ed. by A. Zayats and D. Richard, ArtechHouse, 235-265 (2009).

[2] E. Bründermann, M. Havenith, SNIM-Scanning near-field infrared microscopy, Annu. Rep. Prog. Chem., Sect. C: Phys. Chem., 104, 235 (2008). E. Bründermann, I. Kopf, M. Havenith, Chemical nanoscopy of cell-like membranes, Proc. SPIE 7188 71880I, 1-9 (2009).

[3] K. Meister, D. A. Schmidt, E. Bründermann, M. Havenith, Confocal Raman microspectroscopy as an analytical tool to assess the mitochondrial status in human spermatozoa, Analyst, 135, 1370-1374 (2010).

[4] I. Kopf, C. Grunwald, E. Bründermann, L. Casalis, G. Scoles, M. Havenith, Detection of hybridization on nanografted oligonucleotides using scanning near-field infrared microscopy, J. Phys. Chem. C 114 (2), 1306-1311 (2010).

[5] J.-S. Samson, R. Meißner, E. Bründermann, M. Böke, J. Winter, M. Havenith, Characterization of single diamond-like and polymer-like nanoparticles by mid infrared nanospectroscopy, J. Appl. Phys. 105, 064908 (2009).

[6] M. Filimon, I. Kopf, F. Ballout, D. A. Schmidt, E. Bründermann, J. Rühe, S. Santer, M. Havenith, Smart polymer surfaces: mapping chemical landscapes on the nonometre scale, Soft Matter, (2010) DOI: 10.1039/c0sm00098a 